\title{
Pathway analysis for identification of potential biomarkers in severe cutaneous drug hypersensitivity reactions
}

\author{
Abdallah Ahmed Elbakkoush', Suleman Atique ${ }^{1,3}$, Anas Khaleel ${ }^{2}$ and Chien- \\ Tsai Liu ${ }^{1 *}$ \\ ${ }^{1}$ Graduate Institute of Biomedical Informatics, Taipei Medical University, Taipei 11031, ${ }^{2}$ Institute of Biological Chemistry, \\ Academia Sinica, 128 Academia Road, Section 2, Nankang District, Taipei 11529, Taiwan, ${ }^{3}$ Institute of Public Health, \\ Heidelberg University, 69120 Heidelberg, Germany
}

${ }^{*}$ For correspondence: Email: ctliu@tmu.edu.tw

Received: 19 April 2016

Revised accepted: 15 August 2016

\begin{abstract}
Purpose: To construct a cluster model or a gene signature for Stevens-Johnson syndrome (SJS) using pathways analysis in order to identify some potential biomarkers that may be used for early detection of SJS and epidermal necrolysis (TEN) manifestations.

Methods: Gene expression profiles of GSE12829 were downloaded from Gene Expression Omnibus database. A total of 193 differentially expressed genes (DEGs) were obtained. We applied these genes to geneMANIA database, to remove ambiguous and duplicated genes, and after that, characterized the gene expression profiles using geneMANIA, DAVID, REACTOME, STRING and GENECODIS which are online software and databases.

Results: Out of 193 genes, only 91 were used (after removing the ambiguous and duplicated genes) for topological analysis. It was found by geneMANIA database search that majority of these genes were coexpressed yielding $84.63 \%$ co-expression. It was found that ten genes were in Physical interactions comprising almost $14.33 \%$. There were $<1 \%$ pathway and genetic interactions with values of 0.97 and $0.06 \%$, respectively. Final analyses revealed that there are two clusters of gene interactions and 13 genes were shown to be in evident relationship of interaction with regards to hypersensitivity.

Conclusion: Analysis of differential gene expressions by topological and database approaches in the current study reveals 2 gene network clusters. These genes are CD3G, CD3E, CD3D, TK1, TOP2A, CDK1, CDKN3, CCNB1, and CCNF. There are 9 key protein interactions in hypersensitivity reactions and may serve as biomarkers for SJS and TEN. Pathways related gene clusters has been identified and a genetic model to predict SJS and TEN early incidence using these biomarker genes has been developed.
\end{abstract}

Keywords: Steven-Johnson Syndrome, Toxic epidermal necrolysis, Differentially expressed genes, Hypersensitivity reactions, Genes, Biomarkers

Tropical Journal of Pharmaceutical Research is indexed by Science Citation Index (SciSearch), Scopus, International Pharmaceutical Abstract, Chemical Abstracts, Embase, Index Copernicus, EBSCO, African Index Medicus, JournalSeek, Journal Citation Reports/Science Edition, Directory of Open Access Journals (DOAJ), African Journal Online, Bioline International, Open-J-Gate and Pharmacy Abstracts

\section{INTRODUCTION}

Stevens-Johnson syndrome (SJS) and toxic epidermal necrolysis (TEN) are severe and rare cutaneous adverse reactions (SCAR) manifestations. It is believed that they are caused by certain drugs which may consequently lead to significant morbidity and mortality worldwide [1]. Although, effects of such manifestations are not localized, yet skin is the vulnerable tissue for such reactions. SJS and TEN are considered the most serious and fatal skin diseases among cutaneous drug-induced hypersensitivity adverse reactions [2]. SJS and 
TEN are immune mediated hypersensitivity reactions that involve the skin and mucous membranes. They are distinguished by bullous formation followed by epidermal detachment that eventually affects mucous membranes [3]. Currently, more than $5 \%$ of the patients with SJS and $30 \%$ of the patients with TEN die [4]. However, the underlying mechanism leading to such death have not been discovered yet.

T-cell pathways involvement was reported to be associated with TEN reaction over a decade ago, in addition to the recently reported Granulysin and natural killer cells inhibitory receptors [5-7]. Although these mechanisms were useful to decipher some of the largely unknown mechanisms underlying such a complex disease, yet there must be further predictors and biomarkers on the genetic level. Moreover, various studies have shown that several cytokines might play role in the apoptosis or the cytokine inflammations in SJS and TEN. Among these recently discovered cytokines are the interleukin (IL) IL-6, IL-8 and monocyte chemoattractant protein-1 (MCP-1). All of these were upregulated in such manifestations [8].These cytokines and others are important cellular regulators and mobilizers of cells engaged in innate as well as adaptive inflammatory immunity which has interlocking factors to both SJS and TEN $[9,10]$. Cytokines are released by various cells in the body, usually in response to an activating stimulus, and they induce responses by binding to specific receptors on the surface of target cells [11]. Levels of pro-inflammatory cytokines were significantly higher in SJS and TEN patient samples than the healthy controls [12].

Main objective of the study is to establish relevant biomarkers for SJS and TEN early detection using the genetic mapping of DEG SJS and TEN patients' samples. Protein-protein interaction network and pathways analysis tools along with other online databases and software not only provide information about these specific DEG proteins in severe cutaneous adverse reaction, but also pave the path towards determination of potential early detection biomarkers.

\section{EXPERIMENTAL}

\section{Gene set array}

Gene expression profiles of GSE12829 were downloaded from Gene Expression Omnibus (GEO) database that has been investigated previously by Bellon et al [13]. The samples of twenty three patients suffering from cutaneous drug-induced hypersensitivity reactions were matched to eight healthy controls who were using the same medications. A total of 193 genes were found with distinct expressions in comparison between healthy controls and patients samples. GEO2R technique [14], (which is already available on Gene Expression Omnibus) was used to acquire the relevant gene list. Thereafter, these genes were loaded to geneMANIA database [15] to remove ambiguous and duplicate genes.

\section{Database search}

Gene expression profiles were characterized using DAVID (Database for Annotation, Visualization and Integrated Discovery). DAVID web tool provides a comprehensive set of functional annotation tools for researchers to understand biological meaning behind large list of genes [16]. In this study, the DAVID tool was used to annotate the function of our genes list. It lead to the selection of the Gene Ontology (GO) terms with adjusted $p$-value less than 0.05 and count larger than 5 .

The STRING (Search Tool for the Retrieval of Interacting Genes) version 9.1 [17], database provides both experimental and predicted interaction information. Version 9.1 of STRING covers more than 1100 completely sequenced organisms. To identify the interactive relationships among target genes or other genes, genes list was mapped to STRING. GeneMANIA was again used to figure out genes that have protein and genetic interactions, pathways, coexpression, or co-localization [15]. It was also utilized to generate the relevant network with different scores.

The genes were then analyzed and characterized in KEGG (Kyoto Encyclopedia of Genes and Genomes) pathway categories [18] by the GENECODIS software [19]. REACTOME is a bioinformatics tool for the visualization, interpretation and analysis of pathway knowledge to support basic research, genome analysis, modeling, systems biology and education [20].

\section{RESULTS}

\section{GeneMANIA analysis yielded 9-core nodes genes network}

In this study, multiple databases approaches has been used to identify the pathways and network trends for the produced gene list. Primarily, majority of these genes were co-expressed 
together $84.63 \%$ co-expression, and $14.33 \%$ were in physical interaction. GeneMANIA database [15] search yielded only $<1 \%$ for the pathway and genetic interactions with values of $0.97 \%$ and $0.06 \%$, respectively. The 9 nodes gene network was generated by GeneMania tool [15], as shown in Figure 1.

Main protein-protein interaction networks generated by string (search tool for retrieval of interacting genes) version 9.1 [17]

The protein-protein interaction network for our gene list, was identified using STRING (Search Tool for Retrieval of Interacting Genes) version 9.1 [17]. The result was a generic multiple interacting proteins network, as shown in Figure 2.

After further analysis nine genes with two cluster networks (where most of interacting protein in SJS and TEN present) were focused, and those interacting proteins sub-network (clusters) are shown in Figure 3.
REACTOME pathways analysis results showed 13 gene clusters mainly in immune and signaling pathways

A set of 92 gene was applied to REACTOME pathways database [20]. The results reveal a 13gene cluster that showed that the major pathways were related to immune system 54 (4 $\%)$, signal transduction $76(4 \%)$ and disease pathways $52(5 \%)$.

DAVID; database for annotation, visualization and integrated discovery result is agreeing with REACTOME database

DAVID tool [16] results were also in accordance with the previous REACTOME output. Previous reports have shown $\mathrm{T}$-cell mediated biological processes. DAVID results for the 13 gene were all targeting $\mathrm{T}$-cell namely alpha-beta $\mathrm{T}$-cell receptor complex, T-cell receptor complex, T-cell activation and phosphorylated immune signaling motifs (ITAMS)

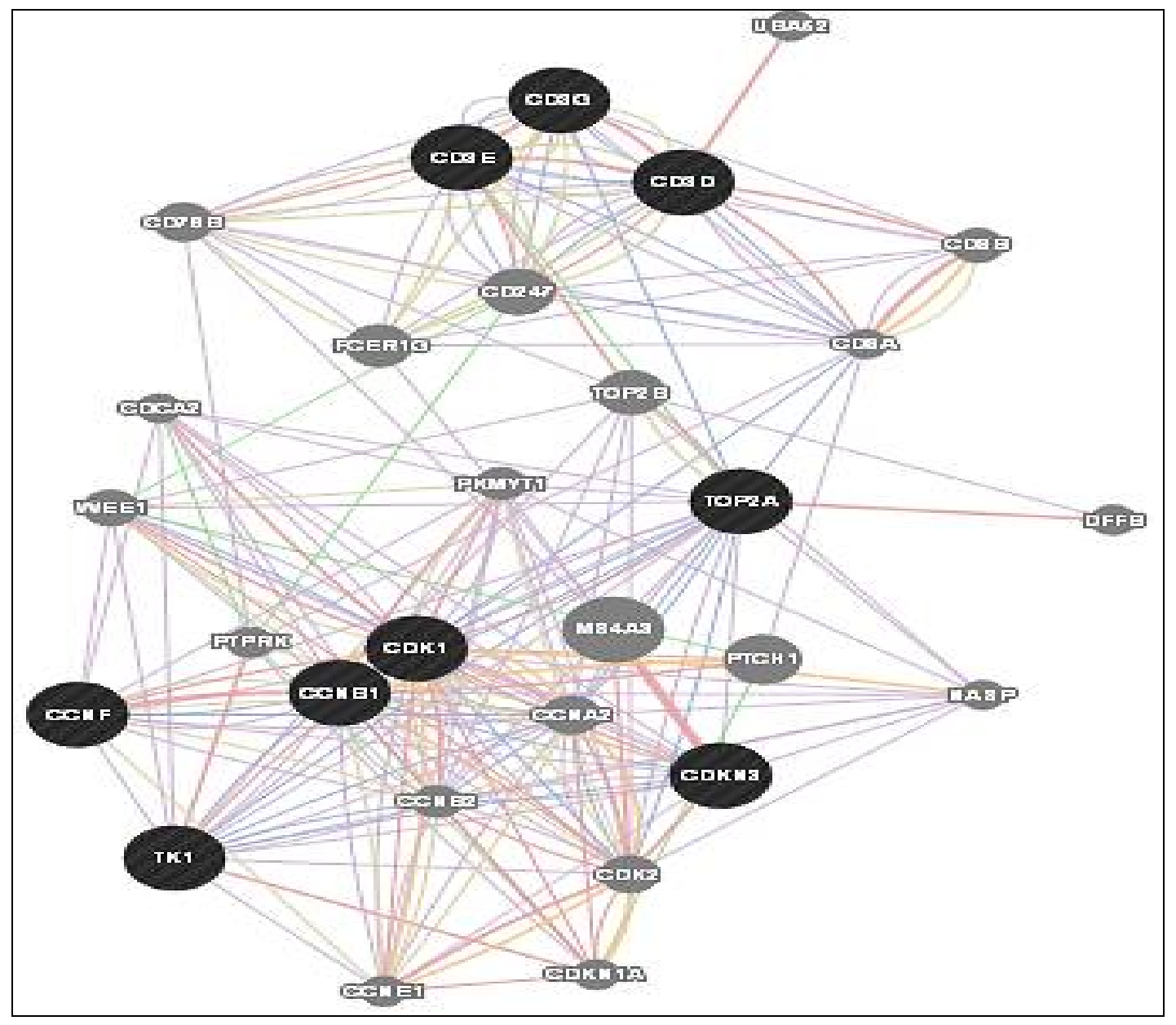

Figure 1: GeneMania primary 9 gene network, black circles indicate the 9 genes core genes 


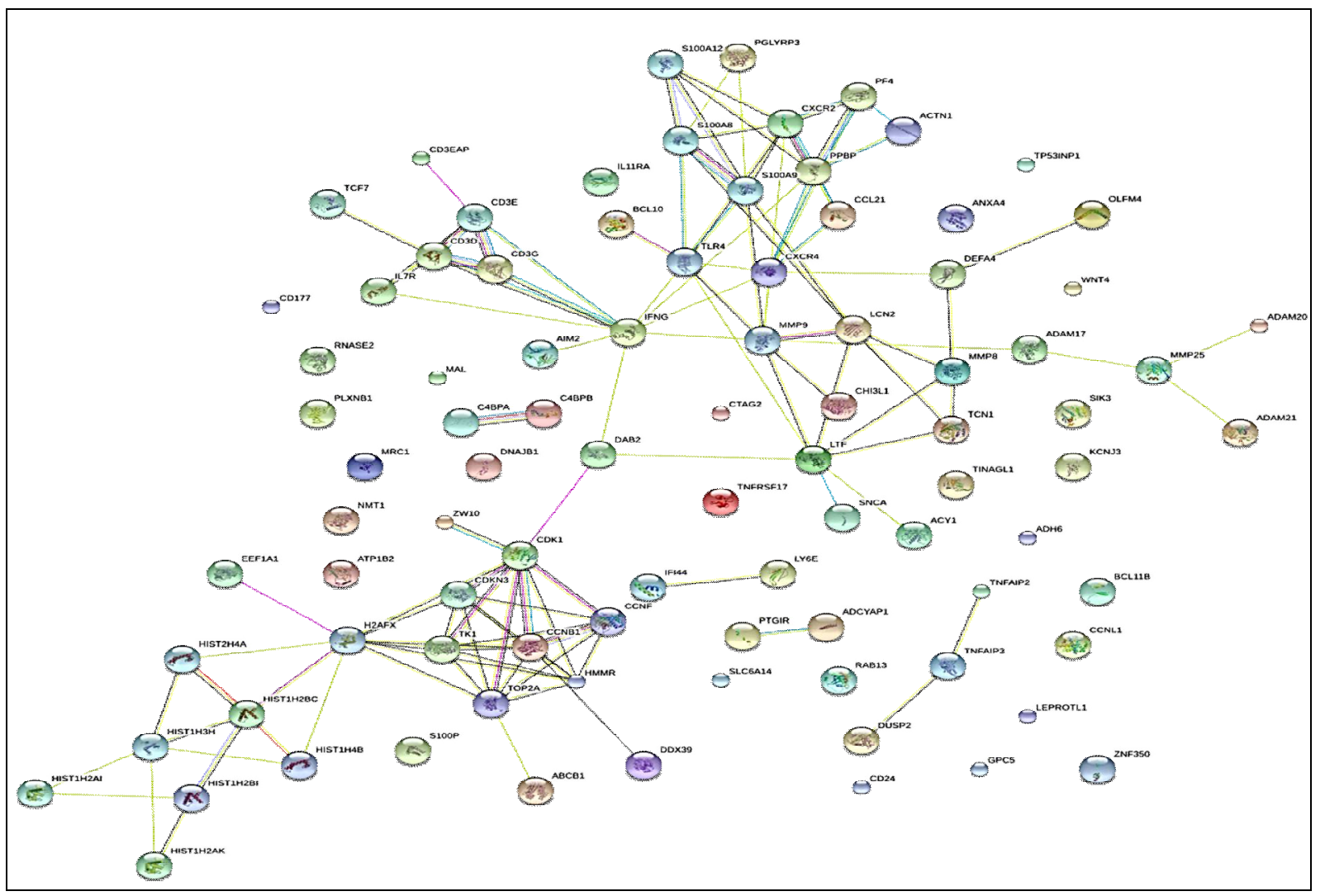

Figure 2: Resulting protein-protein interaction main networks using String 9.1 Web software

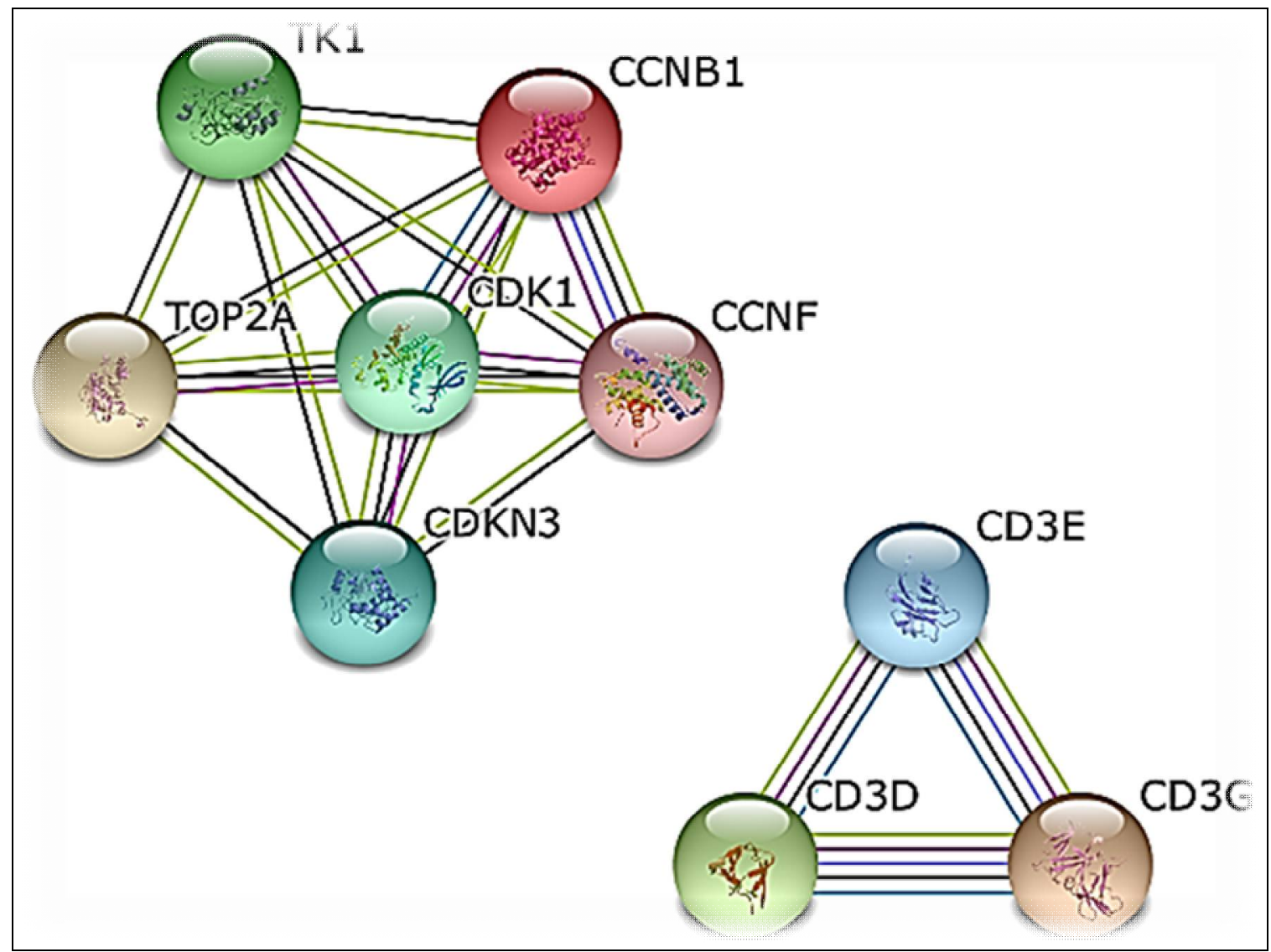

Figure 3: Nine genes which are the core evidence/confidence sub network obtained from STING web tool. It includes: CD3G, CD3E, CD3D, TK1, TOP2A, CDK1, CDKN3, CCNB1 and CCNF 


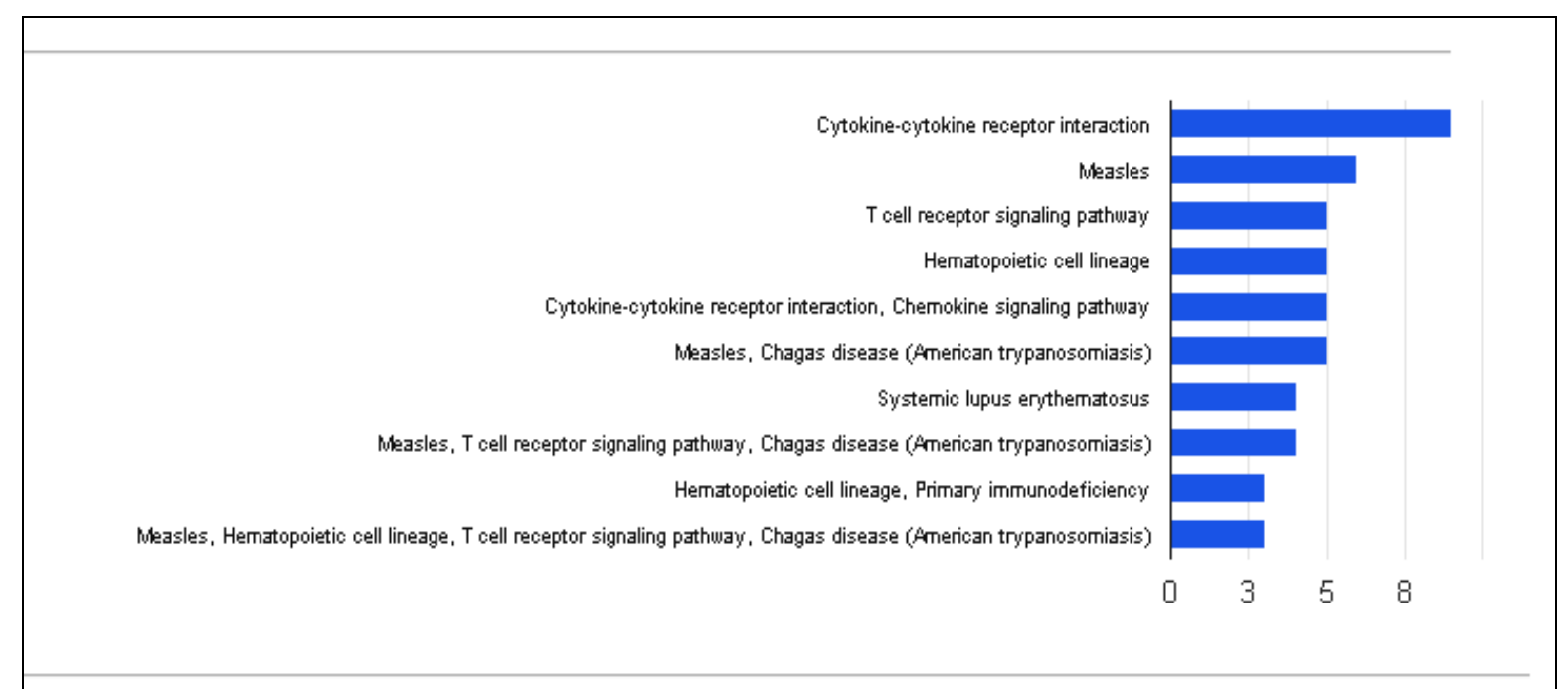

Figure 4: Gene characterization results in the GENECODIS and KEGG pathways tools; ten significantly enriched signaling pathways are shown in descending order of the number of genes contained in each pathway

\section{Protein-protein interaction mainly participated in the biological processes which are related with immune system}

GENECODIS software yielded condensed ten signaling pathways mainly in immune system

The genes were then analyzed and characterized by the GENECODIS program [19]. Ten significantly enriched signaling pathways are shown in descending order of the number of genes contained in each pathway, as explained in Figure 4.

Kyoto encyclopedia of genes and genomes (KEGG) pathway analysis gave two main modules consisting of cytokine-cytokine receptor and $\mathrm{T}$-cell receptor pathways

The results of KEGG pathway enrichment analysis revealed that most of the genes in 2 modules exist in cytokine-cytokine receptor interaction and $\mathrm{T}$ cell receptor signaling pathway. Please refer to supplementary data S5 for further clarification.

Most of these DEG genes were found to be differentially expressed during the early phase of cutaneous drug-induced delayed hypersensitivity reactions. Furthermore, 9 genes were identified with distinct cluster expression and interaction patterns during the acute phase.

\section{DISCUSSION}

This study have shown that specific gene cluster can be used not only for detection of early incidence of SJS and TEN but also to predict clinical prognosis in the same disease setting. Genes and pathways that were associated with incidence of SJS and TEN have been identified via this research. Moreover, it has also been identified that 9 genes signature in SJS and TEN which include: CD3G, CD3E, CD3D, TK1, TOP2A, CDK1, CDKN3, CCNB1, and CCNF. The full name of gene as follows: CD3G, T-cell surface glycoprotein CD3 gamma chain. CD3E, CD3e molecule, epsilon. CD3D, T-cell surface glycoprotein CD3 delta chain. TK1, Thymidine kinase 1. TOP2A, DNA topoisomerase 2-alpha. CDK1, Cyclin-dependent kinase 1. CDKN3, Cyclin-dependent kinase inhibitor 3. CCNB1, G2/mitotic-specific cyclin-B1. CCNF, G2/mitoticspecific cyclin-F.

Up till now, the pathogenesis of SJS/TEN is yet to be fully elucidated. STS and TEN are complex disease forms and could cause certain fatalities. The current study has deployed many online research engines and databases to analyze the differentially expressed genes in these clinical manifestations. geneMANIA results show that many of the inquired genes were ambiguous. However, the majority of these genes have evident role in immune and hypersensitivity reaction mechanisms [21]. Genes pathways in cytokine-cytokine receptor interaction and T-cell signaling shown by KEGG pathway analysis proved that these clusters are directly linked to SJS and TEN disease[18], and then the same has been confirmed via GENECODIS web tool as well [19]. Ten genes showing significantly enriched signaling pathways are shown in descending order of the number of genes contained in each pathway. 
The first candidate pathway was cytokinecytokine receptor interaction which has been validated in many reports to be associated with SJS and TEN manifestations. Previously published studies have shown that several cytokines might play an important role in the apoptosis or cytokine flooded inflammation $[8,22,23]$. Among these recently discovered cytokines are the interleukin (IL), IL-6, IL-8 and monocyte chemo-attractant protein-1 (MCP-1) and all were reported to be unregulated in SJS and TEN [8].

Cytokine-cytokine receptor interaction is the most significant pathway in KEGG and GENCODIS analysis. It is not unusual to find cytokines in SJS and TEN as they are crucial cellular regulators and mobilizers of cells engaged in innate as well as adaptive inflammatory host defense, cell growth, and differentiation aimed at the restoration of homeostasis. Cytokines are released by various cells in the body in response to an activating stimulus, and they induce response by binding to specific receptors on the surface of target cells in case of immunological response as well as in SJS and TEN disease pathophysiology [24].

Previous report by Miyagawa et al [25] state that expression pattern with lower expression of genes coding $T$ cell-specific proteins and high expression of cell cycle-related genes and genes encoding for inflammatory related mediators have been shown [13].

This study extracted the gene set from Bellon et al which has been previously reported as well [13]. The drugs that cause the SJS were Clindamycin $®$, Spiramycin $®$, Statin, Phenytoin $®$ and Allopurinol ${ }^{\circledR}[13]$. These were the trigger of SJS manifestation and from where this gene expressions data come from. Meanwhile, Carbamazepine, furosemide, Erythromycin and paracetamol were more associated with TEN patients in the same cohort [13]. However, there are many other drugs that have shown substantial effects towards provoking SJS and TEN clinical reactions. For instance, in a study conducted by Barvaliya et al [26] it was found that antimicrobials were $50 \%$ associated with these kind of reactions. NSAID's and anti-seizure drugs were also associated and caused $22.41 \%$ and $18.96 \%$ of SJS and TEN cases respectively. According to the mentioned study Nevirapine was the most common drug in this regards which had an association of $28.12 \%$. They also figured out that anti-seizure drugs are mostly associated with such kind of adverse reactions.
Earlier data reports confirm that Han-Chinese subjects who have the $H L A-B^{*} 1502$ allele are highly prone to high risk of SJS and TEN after taking Carbamazepine medication. This allele however has higher prevalence in Asian and especially East Asian populations, including Han Chinese, Filipino, Malay, Indian, and Thai [27]. This proposes importance of gene loci in the investigation of SJS and TEN causal and association studies.

\section{CONCLUSION}

This research highlights 9 genes associated with the early incidence of SJS and TEN. In this study, pathways related gene cluster has also been identified and a model to predict SJS and TEN early incidences has been developed. These findings can be used in assisting and supporting clinical prognosis and diagnostic biomarkers for further investigation and future therapeutic discoveries. It can be suggested that SJS and TEN have certain potential link to genetic grounds which might be helpful for earlier diagnosis of such reactions to avoid more complications leading towards better clinical outcomes.

\section{DECLARATIONS}

\section{Conflict of Interest}

No conflict of interest associated with this work.

\section{Contribution of Authors}

The authors declare that this work was done by the authors named in this article and all liabilities pertaining to claims relating to the content of this article will be borne by them.

\section{REFERENCES}

1. Fernando SL. The management of toxic epidermal necrolysis. Australas J Dermatol 2012; 53(3): 165-171.

2. Saito $N$, Yoshioka $N$, Abe R, Qiao H, Fujita $Y$, Hoshina D, Suto $A$, Kase $S$, Kitaichi $N$, Ozaki $M$, Shimizu $H$. Stevens-Johnson syndrome/toxic epidermal necrolysis mouse model generated by using PBMCs and the skin of patients. J Allergy Clin Immunol. 2013; 131(2): 434441.

3. Harr T, French LE. Stevens-Johnson syndrome and toxic epidermal necrolysis. Chem Immunol Allergy. 2012; 97: 149-166.

4. Yang MS, Kang MG, Jung JW, Song WJ, Kang HR, Cho $\mathrm{SH}$, Min KU. Clinical features and prognostic factors in severe cutaneous drug reactions. Int Arch Allergy Immunol. 2013; 162(4): 346-354. 
5. Nassif A, Bensussan A, Dorothée G, Mami-Chouaib F, Bachot N, Bagot M, Boumsell L, Roujeau JC. Drug specific cytotoxic T-cells in the skin lesions of a patient with toxic epidermal necrolysis. J Invest Dermatol. 2002; 118(4): 728-733.

6. Chung WH, Hung SI, Yang JY, Su SC, Huang SP, Wei CY, Chin SW, Chiou CC, Chu SC, Ho HC, Yang CH. Granulysin is a key mediator for disseminated keratinocyte death in Stevens-Johnson syndrome and toxic epidermal necrolysis. Nat Med. 2008; 14(12): 1343-1350.

7. Le Cleach L, Delaire $S$, Boumsell L, Bagot $M$, Bourgault-Villada I, Bensussan A, Roujeau JC. Blister fluid $T$ lymphocytes during toxic epidermal necrolysis are functional cytotoxic cells which express human natural killer (NK) inhibitory receptors. Clin Exp Immunol. 2000; 119(1): 225-230.

8. Yagi T, Sotozono C, Tanaka M, Fuwa M, Sekiyama E, Ueta M, Tashiro K, Kinoshita S. Cytokine storm arising on the ocular surface in a patient with Stevens-Johnson syndrome. Br J Ophthalmol. 2011; 95(7): 1030-1031.

9. Ueta $M$, Kinoshita S. Ocular surface inflammation mediated by innate immunity. Eye Contact Lens. 2010; 36(5): 269-281.

10. Ueta M. Innate immunity of the ocular surface and ocular surface inflammatory disorders. Cornea. 2008; 27: S31. 40.

11. Zlotnik A, Yoshie O. Chemokines: a new classification system and their role in immunity. Immunity. 2000; 12(2): 121-127.

12. Neuman MG, Cohen L, Nanau RM, Hwang PA. Genetic and immune predictors for hypersensitivity syndrome to antiepileptic drugs. Trans/ Res. 2012; 159(5): 397-406.

13. Bellon T, Alvarez L, Mayorga C, Morel E, Torres MJ, Martín-Díaz MA, Díaz R, Radial A, Carballo M, Blanca $M$. Differential gene expression in drug hypersensitivity reactions: induction of alarmins in severe bullous diseases. Br J Dermatol. 2010; 162(5): 1014-1022.

14. Davis S, Meltzer PS. GEOquery: a bridge between the Gene Expression Omnibus (GEO) and Bio Conductor. Bioinformatics. 2007; 23(14): 1846-1847.

15. Zuberi K, Franz M, Rodriguez H, Montojo J, Lopes CT, Bader GD, Morris Q. GeneMANIA prediction server 2013 update. Nucleic Acids Res. 2013; 41(W1): W115122

16. Dennis G, Sherman BT, Hosack DA, Yang J, Gao W, Lane HC, Lempicki RA. DAVID: database for annotation, visualization, and integrated discovery. Genome Biol. 2003; 4(9): 1.

17. Franceschini $A$, Szklarczyk $D$, Frankild $S$, Kuhn $M$, Simonovic M, Roth A, Lin J, Minguez $P$, Bork $P$, Von Mering $C$, Jensen LJ. STRING v9. 1: protein-protein interaction networks, with increased coverage and integration. Nucleic Acids Res. 2013; 41(D1): D808-815.
18. Kanehisa $M$, Goto S, Sato $Y$, Furumichi M, Tanabe $M$. KEGG for integration and interpretation of large-scale molecular data sets. Nucleic Acids Res. 2011: 1-6.

19. Nogales-Cadenas $R$, Carmona-Saez $P$, Vazquez $M$, Vicente C, Yang X, Tirado F, Carazo JM, PascualMontano A. GeneCodis: interpreting gene lists through enrichment analysis and integration of diverse biological information. Nucleic Acids Res. 2009; 37(suppl 2): W317-322.

20. D'Eustachio P. Reactome knowledgebase of human biological pathways and processes. Bioinformatics for Comparative Proteomics. 2011: 49-61.

21. Warde-Farley D, Donaldson SL, Comes O, Zuberi K, Badrawi R, Chao P, Franz M, Grouios C, Kazi F, Lopes $C T$, Maitland A. The GeneMANIA prediction server: biological network integration for gene prioritization and predicting gene function. Nucleic Acids Res. 2010; 38(supp/ 2): W214-220.

22. Caproni $M$, Torchia $D$, Schincaglia E, Volpi $W$, Frezzolini A, Schena D, Marzano A, Quaglino P, De Simone CL, Parodi A, Barletta E. Expression of cytokines and chemokine receptors in the cutaneous lesions of erythema multiforme and Stevens-Johnson syndrome/toxic epidermal necrolysis. $\mathrm{Br} J$ Dermatol. 2006; 155(4): 722-728.

23. Ang $L P$, Sotozono $C$, Koizumi $N$, Suzuki $T$, Inatomi $T$, Kinoshita S. A comparison between cultivated and conventional limbal stem cell transplantation for Stevens-Johnson syndrome. Am J Ophthalmol. 2007; 143(1): 178-180.

24. Martínez-Cabriales SA, Gomez-Flores M, OcampoCandiani J. News in severe clinical adverse drug reactions: Stevens-Johnson syndrome (SJS) and toxic epidermal necrolysis (TEN). Gac Med Mex. 2015; 151(6): 777-787.

25. Miyagawa F, Hasegawa A, Imoto K, Ogawa K, Kobayashi $N$, Ito $K$, Fujita $H$, Aihara $M$, Watanabe $H$, Sueki $H$, Tohyama M. Differential expression profile of Th1/Th2associated chemokines characterizes Stevens-Johnson syndrome/toxic epidermal necrolysis (SJS/TEN) and drug-induced hypersensitivity syndrome/drug reaction with eosinophilia and systemic symptoms (DIHS/DRESS) as distinct entities. Eur J Dermatol. 2015; 25(1): 87-89.

26. Barvaliya M, Sanmukhani J, Patel T, Paliwal N, Shah H, Tripathi C. Drug-induced Stevens-Johnson syndrome (SJS), toxic epidermal necrolysis (TEN), and SJS-TEN overlap: a multicentric retrospective study. J Postgrad Med. 2011; 57(2): 115-119.

27. Ferrell, P. Brent, and Howard L. McLeod. "Carbamazepine, HLA-B* 1502 and risk of StevensJohnson syndrome and toxic epidermal necrolysis: US FDA recommendations." (2008): 1543-1546. 